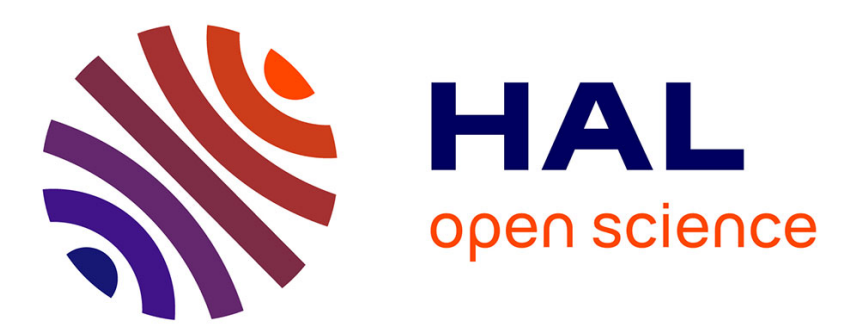

\title{
SNIFS: a wideband integral field spectrograph with microlens arrays
}

\author{
B. Lantz, G. Aldering, P. Antilogus, C. Bonnaud, L. Capoani, A. Castera, Y. \\ Copin, D. Dubet, E. Gangler, F. Henault, et al.
}

\section{- To cite this version:}

B. Lantz, G. Aldering, P. Antilogus, C. Bonnaud, L. Capoani, et al.. SNIFS: a wideband integral field spectrograph with microlens arrays. SPIE 2003, Sep 2003, St Etienne, France. pp.146-155. in2p3-00020373

\section{HAL Id: in2p3-00020373 https://hal.in2p3.fr/in2p3-00020373}

Submitted on 29 Jan 2004

HAL is a multi-disciplinary open access archive for the deposit and dissemination of scientific research documents, whether they are published or not. The documents may come from teaching and research institutions in France or abroad, or from public or private research centers.
L'archive ouverte pluridisciplinaire HAL, est destinée au dépôt et à la diffusion de documents scientifiques de niveau recherche, publiés ou non, émanant des établissements d'enseignement et de recherche français ou étrangers, des laboratoires publics ou privés. 


\title{
SNIFS : a wideband integral field spectrograph with microlens arrays
}

\author{
Blandine Lantz ${ }^{a}$, Greg Aldering ${ }^{b}$, Pierre Antilogus ${ }^{c}$, Christophe Bonnaud $^{a}$, Lionel Capoani ${ }^{a}$, \\ Alain Castera $^{d}$, Yannick Copin ${ }^{d}$, Dominique Dubet ${ }^{a}$, Emmanuel Gangler $^{d}$, François Hénault ${ }^{a}$, \\ Jean-Pierre Lemonnier ${ }^{a}$, Raynald Pain ${ }^{c}$, Arlette Pécontal ${ }^{a}$ and Emmanuel Pécontal ${ }^{a}$ \\ a Centre de Recherche Astronomique de Lyon, France \\ 6 Lawrence Berkeley National Laboratory, USA \\ ${ }^{c}$ Laboratoire de Physique Nucléaire et de Hautes Energies, France \\ ${ }^{d}$ Institut de Physique Nucléaire de Lyon, France
}

\begin{abstract}
SNIFS is an integral field spectrograph devoted to the observation of supernovae. This instrument is today in the manufacturing phase and should be able to observe supernovae at the end of this year (2003) on the 2.2m telescope of University Hawaii.

The concept of SNIFS is to split the 6" $\mathrm{x} 6$ " field of view into 225 samples of 0.4 " $\mathrm{x} 0.4$ " through a microlens array. Then the spectral decomposition of each sample is imaged on a $2 \mathrm{k} x 4 \mathrm{k}$ CCD. In order to cover all the large spectral range with a high resolution, the spectrograph is composed of two modules, one for the blue wavelengths $(320 \mathrm{~nm}$ to $560 \mathrm{~nm}$ ) with a resolution around 1000 at $430 \mathrm{~nm}$ and one for the red wavelengths (520 nm to $1 \mu \mathrm{m})$ with a resolution around 1300.at $760 \mathrm{~nm}$.
\end{abstract}

First we will present the optical design and detail the function of each optical component. Then the mechanical design will be shown with some maps of the structure. Finally the first pictures taken during the alignments will be displayed.

Keywords: Integral Field Spectroscopy, Microlens array, SNIFS, UH, supernovae

\section{INTRODUCTION}

SNIFS (SuperNovae Integral Field Spectrograph) is an astronomical instrument dedicated to the spectrography of relatively nearby supernovae, with a redshift around 0,07. This instrument is the cornerstone of the Nearby Supernovae Factory project (e.g. Aldering and al. $2002^{[1]}$, Pécontal and al. $2003^{[2]}$ ) which aim is to produce a large sample (around 300 ) of type Ia supernovae, followed spectrophotometrically during the 40 first days of their evolution. We want to obtain simultaneously the spectrum of a supernova and its galaxy in order to subtract the continuous background of the galaxy from the spectrum of the supernova. The spectral range covers a large band from $320 \mathrm{~nm}$ to $1 \mu \mathrm{m}$ to provide as much information as possible on the characteristics of the supernovae.

After TIGRE, SAURON and OASIS (e.g. Bacon and al. $1995^{[3]}$ ), SNIFS is the fourth integral field spectrograph built by the Lyon Observatory. This kind of instrument provides the spectral decomposition of light in one unique observation on all the field of view. Compared with a classic spectrograph where a slit scans the field, the exposure time is considerably reduced and the variation of the atmospheric turbulence does not disturb the results any more. These four instruments are all based on the same optical concept: a microlens array is used to slit up an enlarged image of the telescope focus into several spatial samples. Then a classical spectrograph, using a grating, makes an image of the spectral decomposition of each sample on a CCD. The particularity of these instruments is that after the microlens array, all spatial information is lost. All the rays bellowing to the same spatial sample are focused on the same spectrum on the CCD. This is due to the inversion between the field and the pupil in the plane of the microlens array. 


\section{OPTICAL DESIGN}

\subsection{The optical requirements}

The objects observed by SNIFS will be supernovae, that means point sources. As the instrument will be mounted on the $2.2 \mathrm{~m}$ University Hawaii telescope without adaptive optic, the image of a supernova will be limited by the seeing, which mean value is about 0.8 ". The spatial sampling of SNIFS is 0.4 "x0.4"; a supernova is then represented by a minimum of four samples.

Concerning the detectors, each one is composed of 2048x4096 pixels of 15 microns. For the blue channel, the detector is an E2V thin CCD; whereas two deep depleted E2V detectors (40 $\mu \mathrm{m}$ thick) are used for the guiding and the photometric channel. Concerning the red spectrograph, the Lawrence Berkeley National Laboratory is developing a thick deep depleted detector $(300 \mu \mathrm{m}$ thick) which will be used for this channel but it does not work perfectly well yet. However, another E2V deep depleted detector ( $40 \mu \mathrm{m}$ thick) is available and should substitute the LBNL detector for the beginning of the instrument's life.

A compromise has been reached between the size of the field of view and the spectral resolution so that all the spectra fit onto the detector. The resolution is around 1000 and the full field of view covers 6"x6". The width of the spectra must be of course as small as possible, not to overlap the adjacent spectrum. A requirement of 2 pixels is chosen for this width.

The use of a grating in the spectrograph limits the spectral bandwidth to an octave, whereas SNIFS spectral range covers nearly two octaves. The solution chosen is to split the beam in two channels through a dichroic mirror. The blue channel begins at $320 \mathrm{~nm}$ and ends at $560 \mathrm{~nm}$ while the red channel explores from $520 \mathrm{~nm}$ to $1 \mu \mathrm{m}$. Both channels cover less than one octave and there is $40 \mathrm{~nm}$ overlapped between the two channels.

Table 1 shows the main parameters of SNIFS:

\begin{tabular}{|l|l|}
\hline Item & Requirement \\
\hline \multirow{3}{*}{ Field of view } & 6 " x 6" \\
& $658 \times 658 \mu \mathrm{m}$ at focal plane of the telescope \\
& $21 \times 21 \mathrm{~mm}$ at microlens array plane, size of the microlens array \\
\hline Enlarger magnification ratio & -31.9 \\
\hline & $0.4 " \times 0.4 "$ \\
Spatial sampling & $44 \times 44 \mu \mathrm{m}$ at focal plane of the telescope \\
& $1.4 \times 1.4 \mathrm{~mm}$ at microlens array plane, size of one microlens \\
& $(15 \times 15$ spatial samples, micro lenses $)$ \\
\hline Spectrograph magnification ratio & -1.35 \\
\hline & 320 to $560 \mathrm{~nm}:$ \\
& $0.22 \mathrm{~nm} / \mathrm{pixel}$, resolution around 1000 at $430 \mathrm{~nm}$ \\
\hline Spectral sampling & 520 to $1000 \mathrm{~nm}:$ \\
& $0.3 \mathrm{~nm} / \mathrm{pixel}$, resolution around 1300 at $760 \mathrm{~nm}$ \\
\hline \multirow{2}{*}{ CCD dimensions } & $2048 \times 4096$ pixels of $15 \mu \mathrm{m}$ \\
\hline & $30.72 \times 61.44 \mathrm{~mm}$ \\
\hline
\end{tabular}

Table 1: Characteristics of SNIFS

The next paragraphs detail the main components of the instrument: the enlarger, the dichroïc mirror, the microlens array and the spectrograph. In addition, a calibration unit as well as a guiding and a photometric channel are present on the instrument and will be rapidly presented. Nearly all the optic components of SNIFS have been manufactured by the French company SESO. 


\subsection{The enlarger and the dichroïc mirror}

Due to the characteristics of the UH telescope, the square field of view of 6" 6 " correspond to a physical size of $658 \times 658 \mu \mathrm{m}$ at the telescope focus. The present technology rules out the direct sampling of this field as a step of 0.4 " would correspond to a lenslet size of $44 \mu \mathrm{m}$. The aim of the enlarger is therefore to expand the field of view up to a reasonable size $(21 \times 21 \mathrm{~mm})$, where the microlens array can be placed. This function is realised with a simple doublet, called the enlarger doublet, whose object focus is just near the telescope focus. As a consequence the image is far away with respect to the magnification ratio of the enlarger.

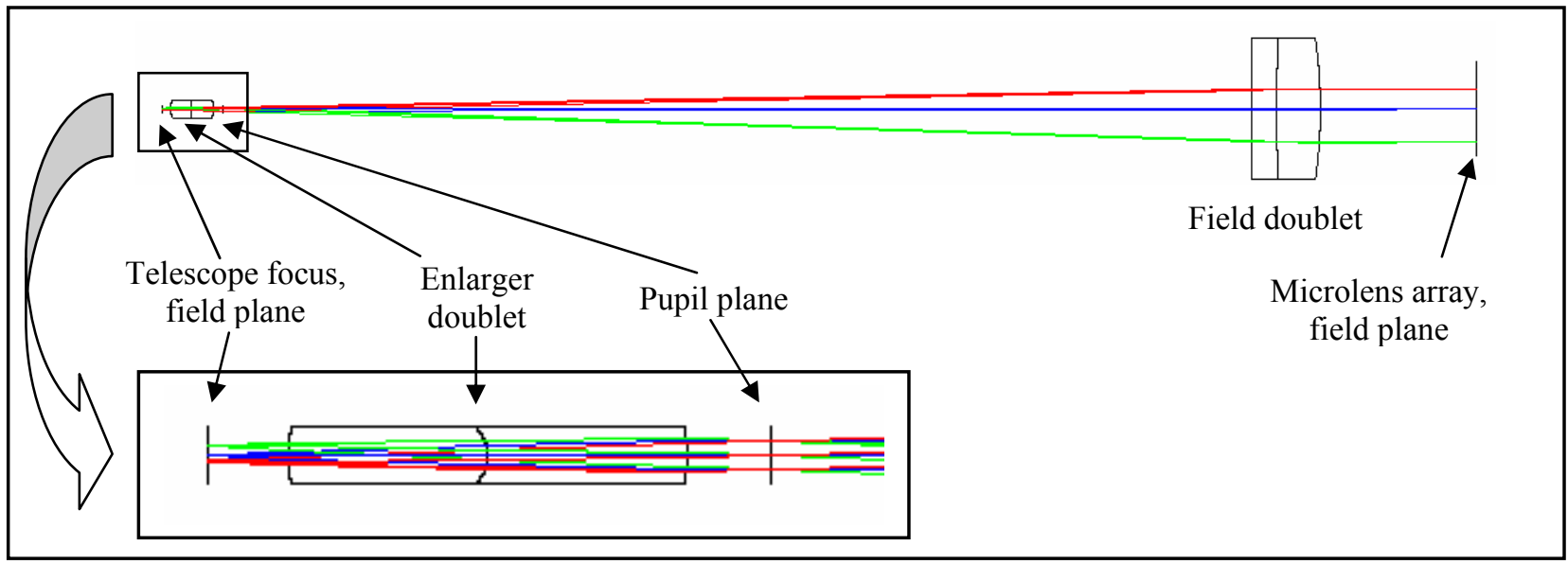

Figure 1: Optical layout of the enlarger

The second function of the enlarger is to produce a telecentric beam at the entrance of the microlens array. The pupil must be at infinite distance in this plane. In this way, for each microlens the beam is at normal incidence and the conjugation is the same whichever lens we consider. The entrance pupil of the instrument is the primary mirror of the telescope. As the entrance pupil is very far in front of the enlarger doublet, we find an intermediate image of the pupil at the image focus of the doublet EL. To put the pupil to infinity on the microlens array we just need a doublet, called field doublet, with its object focus on this intermediate pupil plane. This doublet is placed near the array, not to affect the field conjugation. The figure 1 shows the optical layout of the enlarger.

The image quality of the enlarger is limited by the diffraction as shown on the figure 2: the spot diagrams are smaller than the Airy disk. Concerning the pupil, the telecentricity is better than $0.2^{\circ}$.

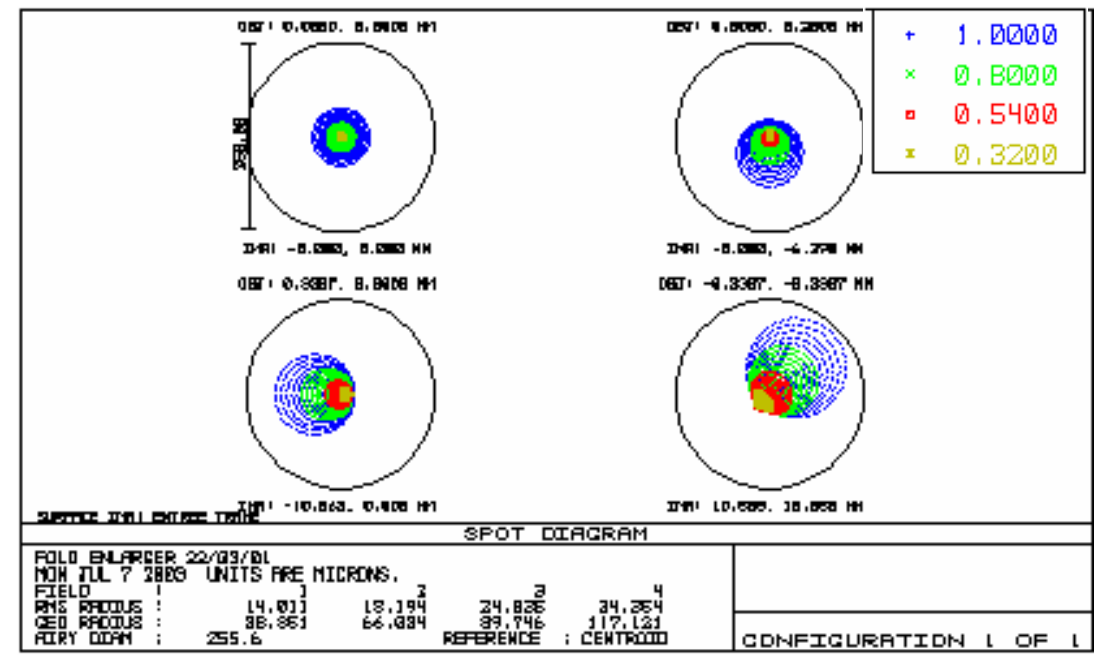

Figure 2: spot diagram for the enlarger, the circle represent the Airy diameter for the smallest wavelength 
A dichroïc mirror is placed between the enlarger doublet and the field doublet to split the spectral range in two channels. The blue wavelengths are reflected against the dichroïc whereas the red wavelengths are going through the dichroïc. A colored glass in the back of the dichroïc blocks the residual blue wavelengths in the red channel. After the dichroic, the field doublet and the microlens array are optically identical for both channels, differing from each other in their antireflection coating only. Of course the instrument includes two spectrographs but they are not similar because they are optimized for different wavelength ranges. The figure 3 shows the characteristics of the dichroïc.

\section{Characteristics of the dichroïc mirror}

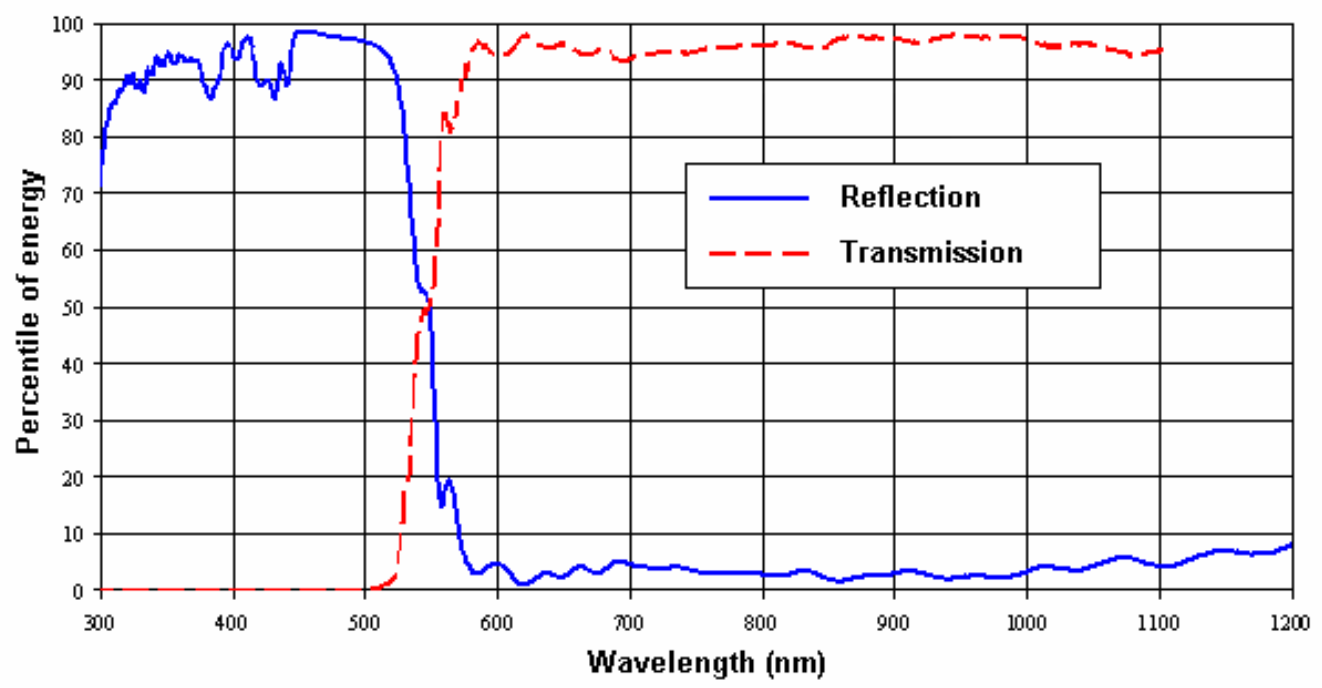

Figure 3: performances measured on the manufactured dichroïc mirror

\subsection{The microlens array and the inversion between field and pupil}

The supernova and the galaxies we want to observe are focused on the microlens array. In this plane the array provides a sampling of the image. The field is split into 225 squared samples $(25 \times 25)$. Concerning the pupil, the beam is telecentric at the entrance of the microlens array, therefore an image of the pupil can be found at the focus of each micro lens. Due to the small focal length of the micro lenses $(6.67 \mathrm{~mm})$ and the high aperture number $(\mathrm{F} / 325)$ the sub-pupils measure only $22 \mu \mathrm{m}$ at the focus plane of the microlens array. Then the spectrograph images the spectral decomposition of these small sub-pupils on the detector but not the spectral decomposition of the field. We don't care any more about the field conjugation; there is an inversion between the field and the pupil. This means that the spatial resolution is limited by the size of the micro lenses. All the fields hitting the same micro lens will generate the same footprint on the pupil at the focal plane of the micro lens. Therefore the spectrum on the detector, which is the dispersed image of the pupil, will be the same for all these fields. The optical path for different fields is represented on the figure 4.

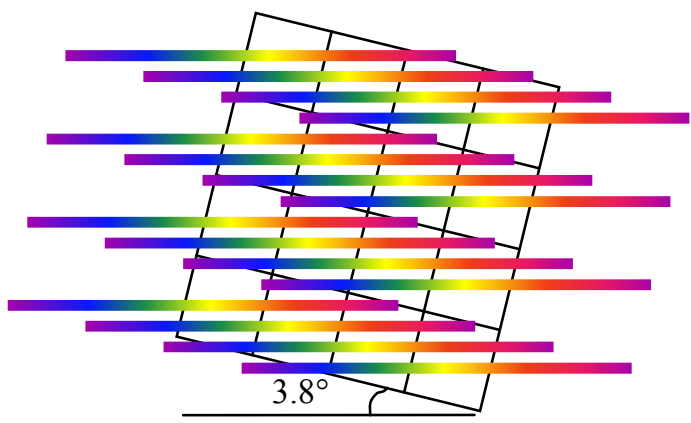

Figure 5 : Tilt of the microlens array around the optical axis 
3 fields focused on the same microlens array with a high depth of field, they are images of the telescope focus plane

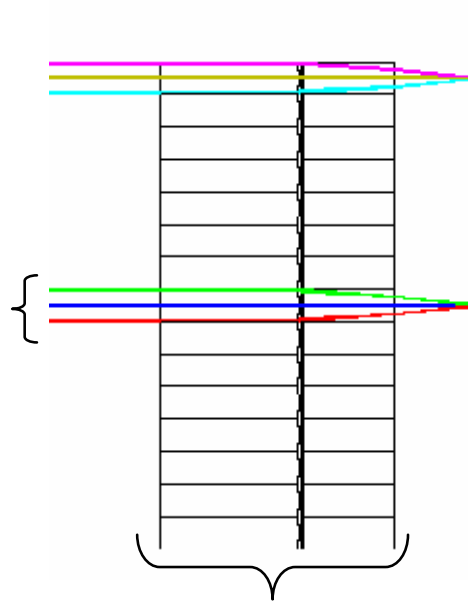

Microlens array, composed of two cylindrical lenses
2 sub-pupils which are objects for the spectrograph

Focal plane of the microlens array

Figure 4: optical layout of the microlens array

As all the micro lenses generate a long spectrum of a thousand of pixels, the microlens array needs to be tilted around the optical axis to avoid any overlap between the spectra. In addition the zero order and the second order of the grating, which are both less bright than the first order, should not pollute the useful spectra. To distribute well all the spectra on the detector, an angle of $3.8^{\circ}$ must be introduce between the microlens array and the dispersive direction. The figure 5 explains this rotation of the microlens array.

Concerning the manufacture of the microlens arrays, each micro lens is composed of two crossed cylindrical lenses with an air space of $100 \mu \mathrm{m}$ between the two lenses. The manufacturer of this special kind of microlens array is the German company LIMO. The figure 6 is composed of a scheme of the crossed cylindrical lenses and a photo is added to illustrate.
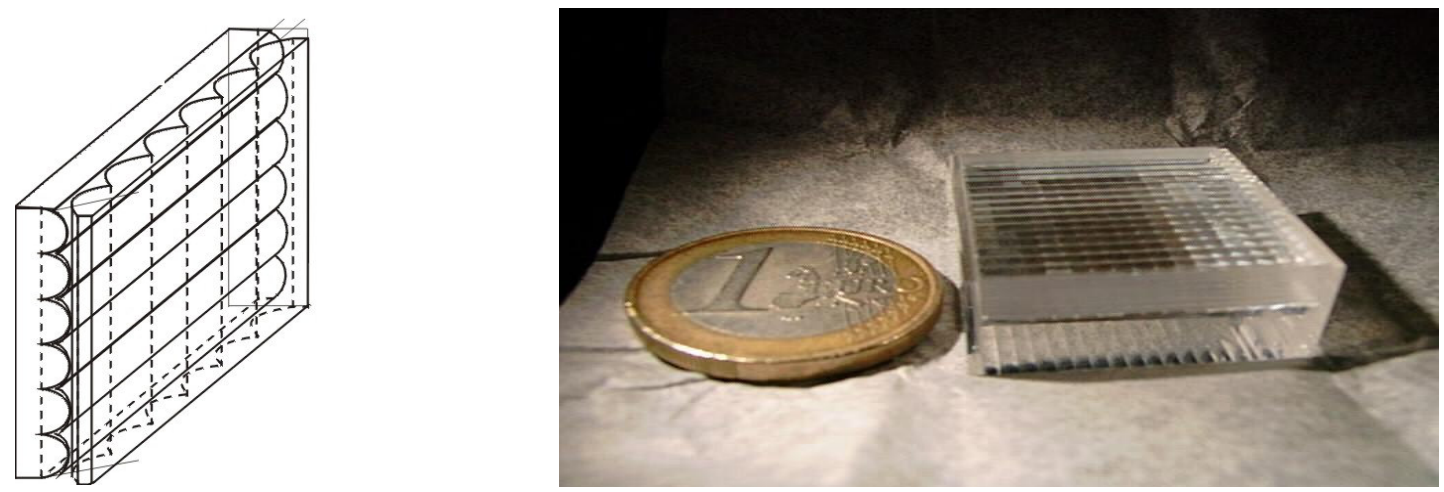

Figure 6 : A microlens array

\subsection{The spectrograph}

The two spectrographs are based on the same principle. The sub-pupils at the focal plane of the microlens array are placed at the object focal plane of a collimator. The parallel beam which comes out from the collimator goes through the dispersive element before being refocused on the detector by a camera. In order to compact the instrument, the beam is bent between the collimators and the cameras. This rotation of $100^{\circ}$ is produced by an internal reflection in a prism. Then a GRISM (grating-prism) is added against this prism, it provides in-line viewing for the central wavelength of the spectral range. To reduce the size of the grating, the pupil is focused on the grating as shown of the figure 7 . 


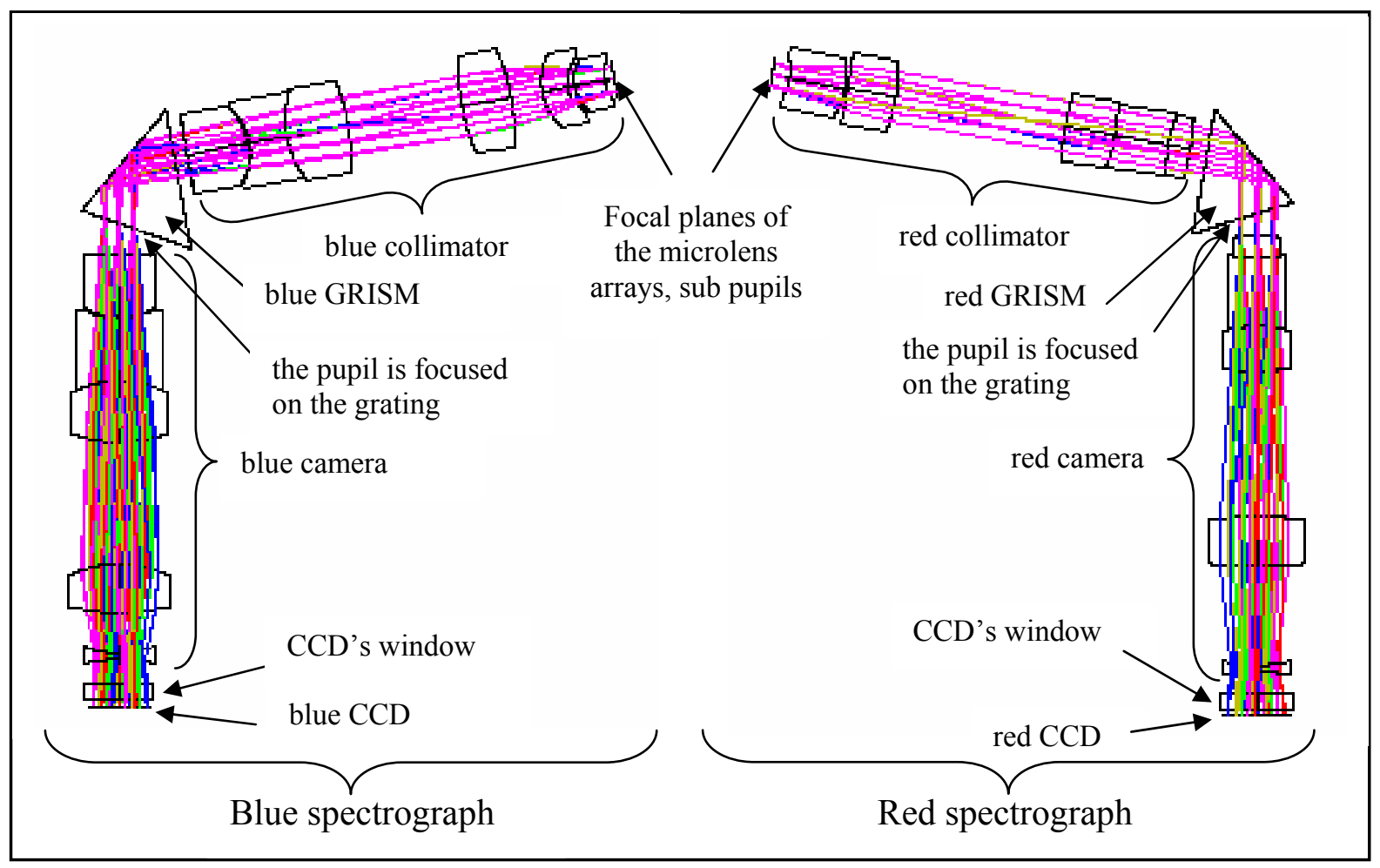

Figure 7: layout of the two spectrographs

\subsection{The calibration unit and the guiding and photometric channel}

Science exposures will generally have a poor signal to noise ratio. Thus, to calibrate the position of the spectra on the detector, an integrating sphere simulates the primary mirror of the telescope illuminated homogeneously. Two doublets are used to image the output of the integrating sphere to the image focus of the enlarger doublet. The calibration unit also allows us to correct the non-uniformity of the microlens array and of the CCD. The sphere is used with either continuum lamps or spectral lamps (argon and mercury-cadmium) so that both red and blue channels can be calibrated. A calibration exposure is done before each scientific exposure in order to take account of the flexures of the instrument. If needed a second calibration exposure will be done after the scientific exposure but, as the scientific exposure time is only around half an hour, the calibration exposure shouldn't move during this short time.

A pick off prism is placed between the telescope and the enlarger. The beam is bent by an internal reflection in the prism from the telescope axis to the plane of the flange of the instrument. When the prism is removed the calibration unit can be used. Directly behind the pick off prism, in the same direction than the telescope axis; two CCD are positioned at the focus of the telescope. One is used for the guiding and the other one is dedicated to a photometric survey. Different filters can be inserted before these detectors.

\section{MECHANICAL DESIGN}

Supernovae are transient objects and they must be observed as soon as they are detected. The instrument need then to be mounted permanently on a telescope. SNIFS will be installed on the south bent Cassegrain focus of the University Hawaii telescope. Moving from this focus to the direct cassegrain one is very fast, needing only a mirror motion. The total mass and the stability of the instrument have been the main driving points for the mechanical design. In order to compact the instrument, the optical axis has been bent several times so that the final size of the aluminium flange is $1.5 \mathrm{~m}$ $\mathrm{x} 1 \mathrm{~m}$. The total mass of the instrument will be around $260 \mathrm{~kg}$ with some additional mass deported from the flange for the calibration lamps and for the electronic $(100 \mathrm{~kg})$. Two pictures of the mechanical design can be found in figure 8 and 9 . The light is coming from behind in both view. 


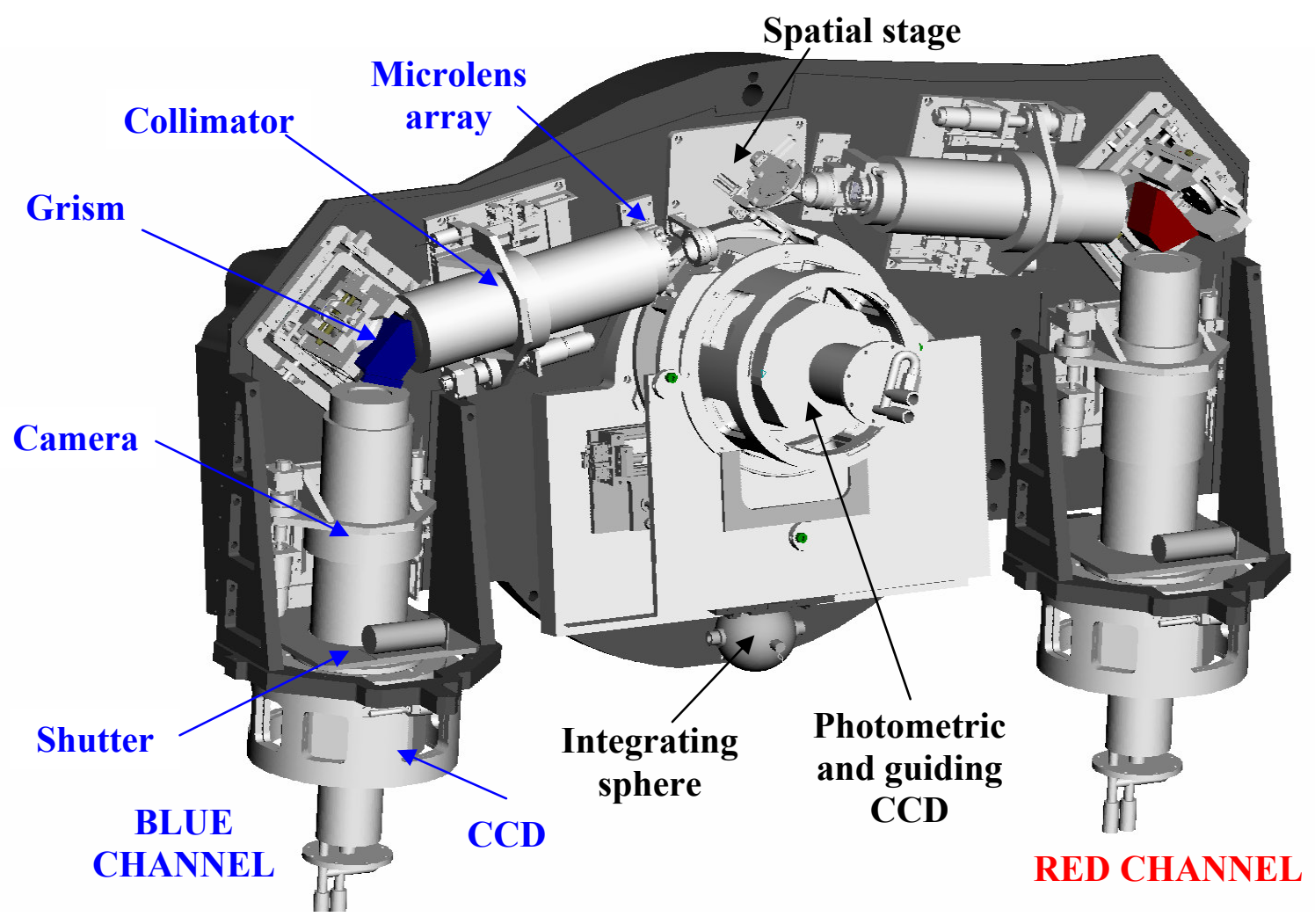

Figure 8: general mechanical design of the instrument

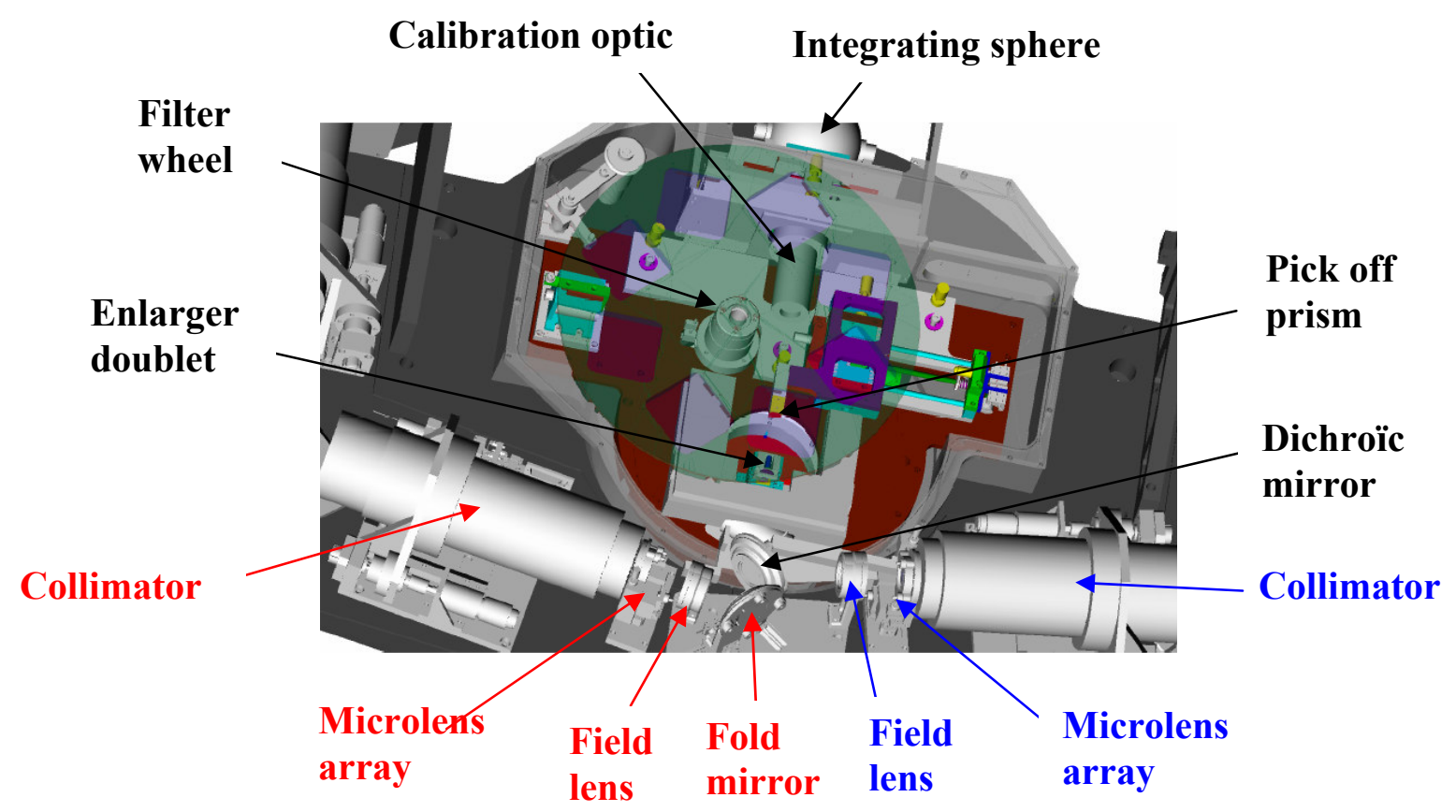

Figure 9: zoom on the central part of the instrument 
As the instrument moves with the telescope during an exposure, the flexion of the instrument must be contained. The flange has been studied with finite element analysis; each component fixed on the flange has been modelled for this analysis with its right weight. The conclusion shows that the maximum amplitude of the deformation does not exceed $5 \mu \mathrm{m}$ for an exposure time of half an hour. The two spectral detectors are the most sensitive components due to their weight $(8 \mathrm{~kg})$.

In order to perform good alignments, nearly all the mechanical mounts provide some precision adjustments. A motorized motion along the optical axis is available on the blue collimator and on the red camera. These adjustments are used to refocus both spectrographs in step with the temperature variations. At the summit of Mauna Kea the temperature varies indeed from $0^{\circ}$ to $5^{\circ} \mathrm{C}$. These variations introduce some expansion of the mechanical mounts and of the lenses, in addition the refractive index of the glasses changes with the temperature. A thermal analysis shows that a refocus of the blue collimator and of the red camera provides a good image quality for this temperature range.

\section{THE OPTICAL ALIGNMENT}

The alignment of SNIFS is done is three steps. First, each spectrograph is adjusted and focused separately from the rest of the instrument, then the calibration unit and the enlarger are aligned. By that time some calibration exposure can be performed. The last step is to control the confocality between the spectrometric channels and the photometric channel. The picture 10 shows the instrument during the alignments.

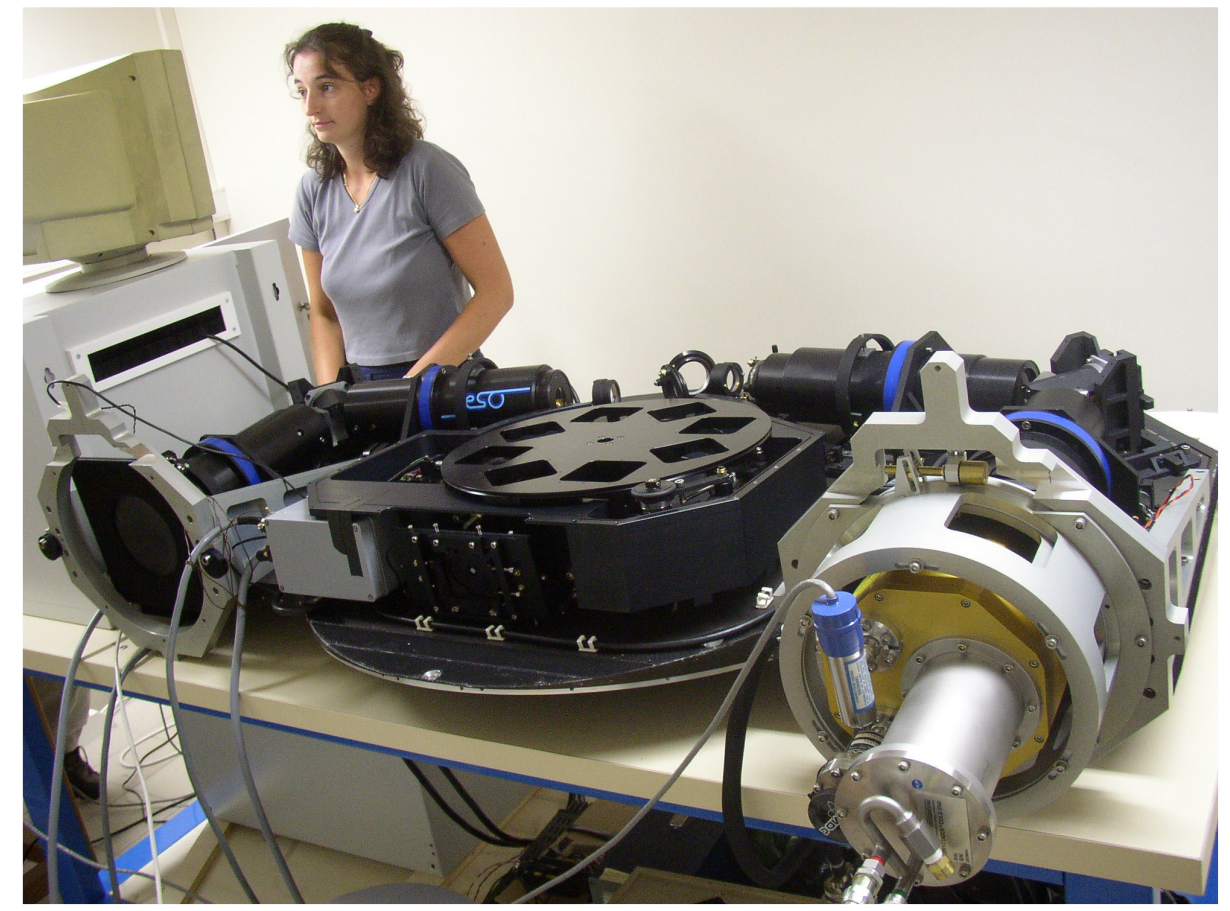

Figure 10: photo during the alignments

The most critical step is the alignment of both spectrographs. The microlens array, the collimator, the GRISM, the camera and the CCD are first tilted in order to merge exactly their optical axis. These adjustments are achieved by autocollimation on the plane surfaces for the CCD, the GRISM and the microlens array or by autocollimation on the center of curvature of the dioptres for the collimator and the camera. With the aim of focusing the spectrograph, a spectral lamp illuminates a hole at the object focus of the field doublet of the enlarger. This beam simulates precisely the working configuration of the instrument when the size of the hole corresponds to the size of the pupil at this plane. We just need to adjust the position of the collimator and of the camera along the optical axis in order to find the best focus of the spectrograph. 
The figure 11 shows a zoom on three lines of micro lenses for the red spectrograph. The exposure was done with a spectral mercury-cadmium lamp. Each point on the detector represents one wavelength for one micro lens. The image quality measured is already acceptable; the full width at half maximum varies between 1.8 and 2.3 pixels for the different micro lenses and the different wavelengths. With a continuum lamp we can verify that the spectra do not overlap. The figure 12 represents the same part of the detector than the figure 11 but the calibration exposure is done with such a continuum lamp. We can easily identify the different order of diffraction of the grating on this picture. Only the first order is useful, the zero order and the second order are pollution. A cross-section from figure 12 in the cross dispersion direction is plot on figure 13 .

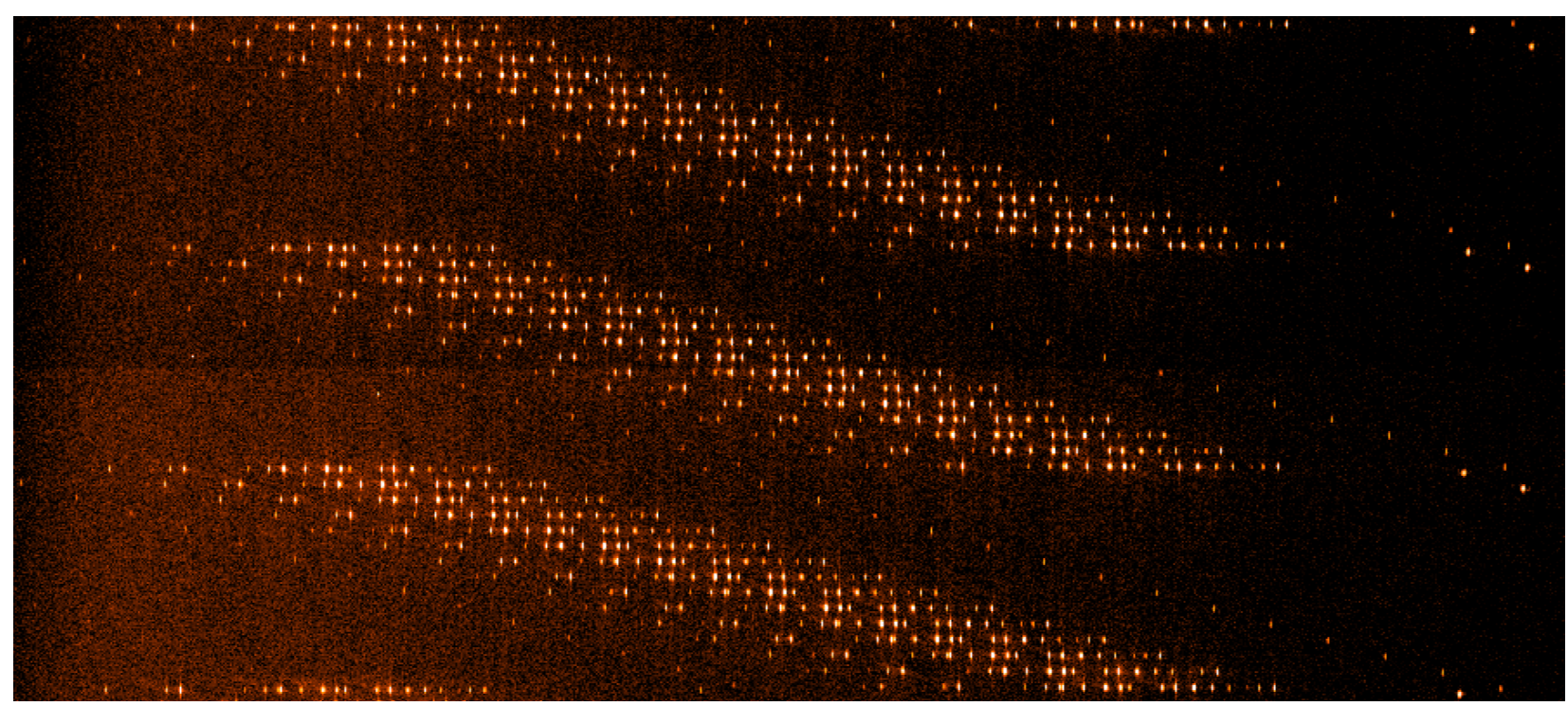

Figure 11: calibration exposure with argon lamp

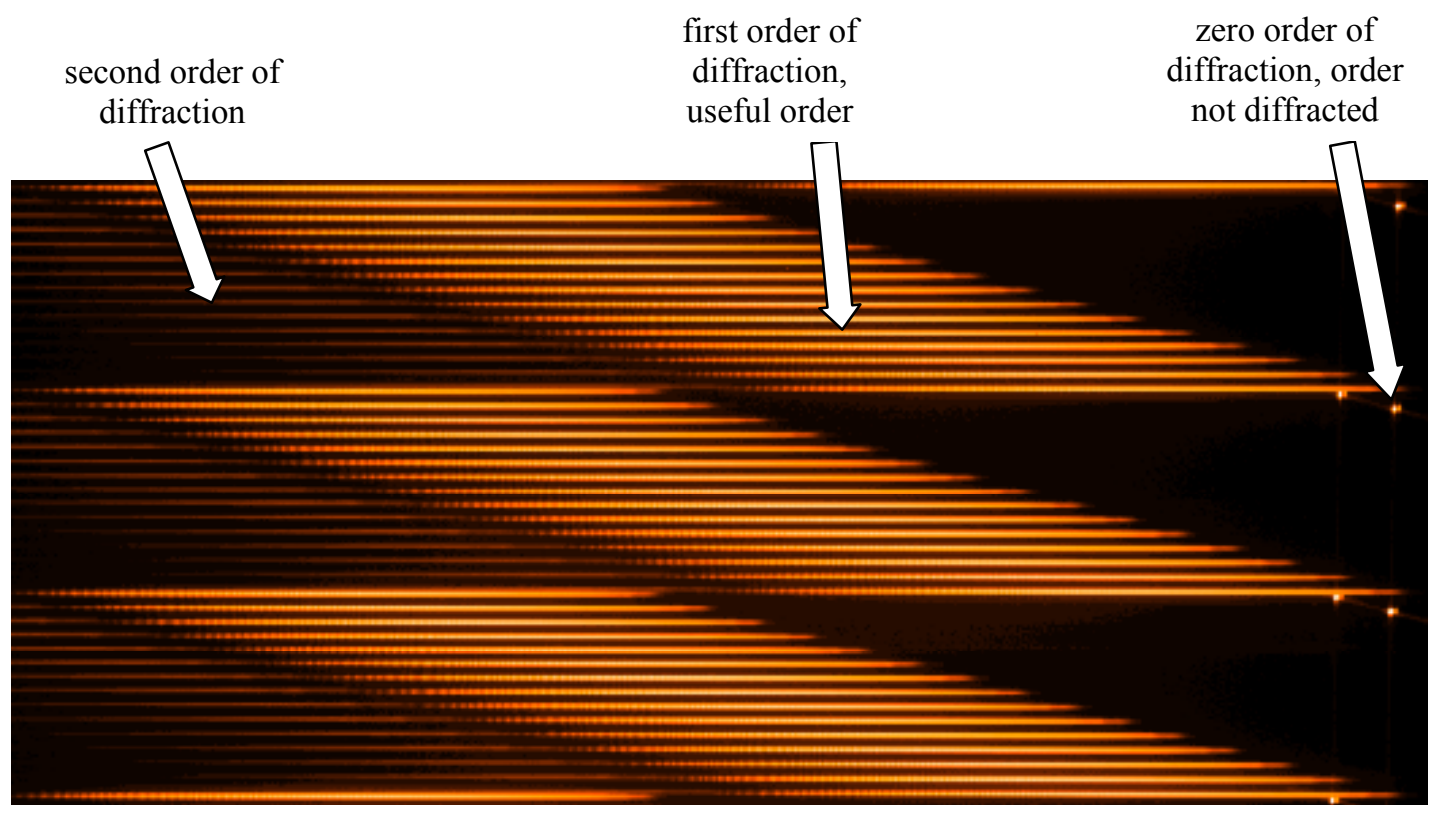

Figure 12: calibration exposure with continuum lamp 


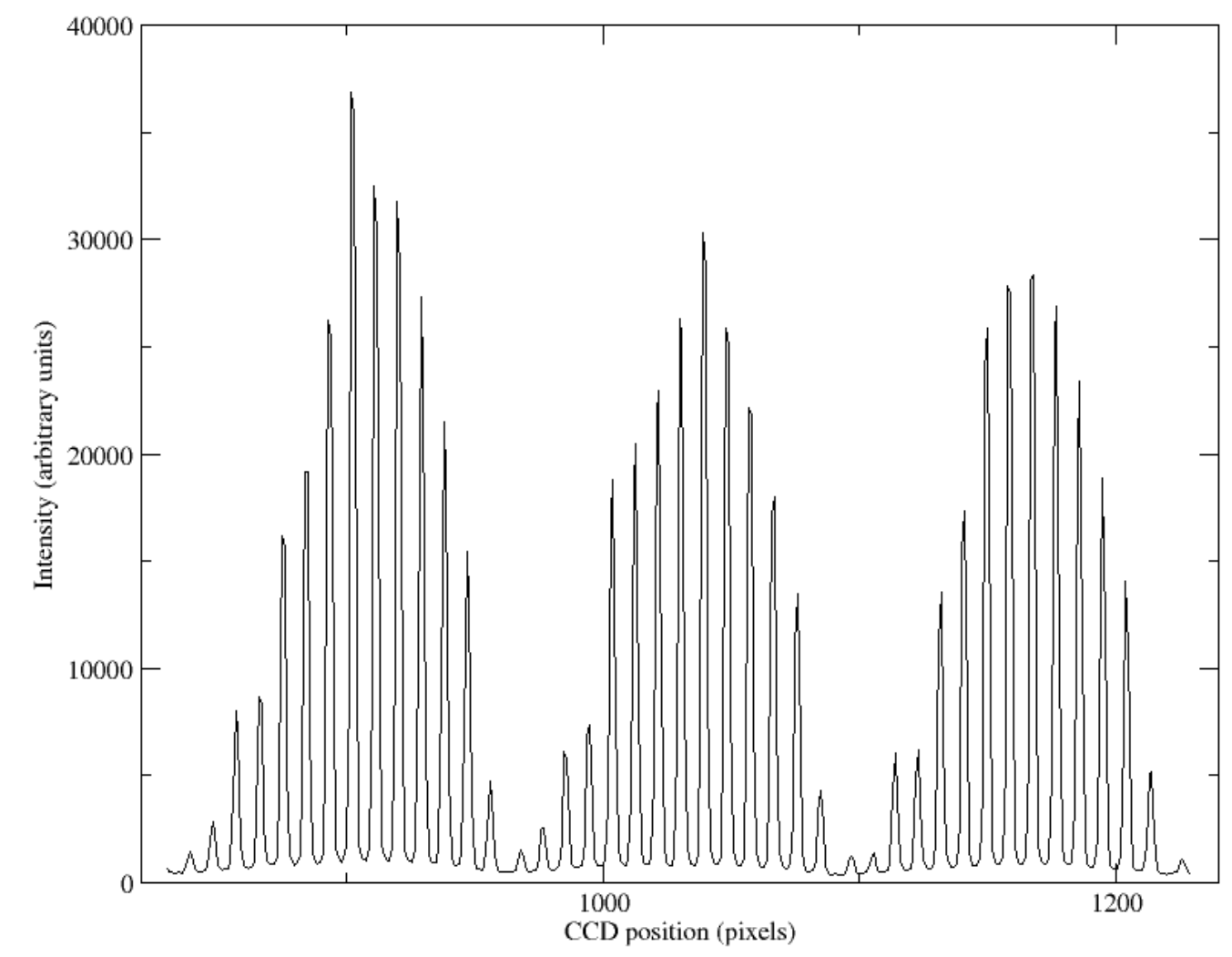

Figure 13: cross-section in the cross dispersion direction for a calibration exposure with a continuum lamp

\section{CONCLUSION}

The optical design of SNIFS has been presented. The very large spectral range obliges us to build two different spectrographs, one for the blue and the other one for the red. The inversion between the field and the pupil in the plane of the microlens array create a spatial sampling. Concerning the mechanical design, the stability of the instrument has been taken into account from the beginning of the study, that's why the instrument should be very stable. The first calibration exposures obtained during the alignment of SNIFS are promising. With such a large spectral range this instrument will permit the scientific community to learn a lot about supernovae which are presently the best tools to measure the expansion of the universe.

\section{REFERENCES}

1. G. Aldering, G. Adam, P. Antilogus, P. Astier, R. Bacon, S. Bongard, C. Bonnaud, Y. Copin, D. Hardin, F. Henault, D. Howell, J.P. Lemonnier, J.M. Levy, S. Loken, P. Nugent, R. Pain, A. Pecontal, E. Pecontal, S. Perlmutter, R. Quimby, K. Schahmeneche, G. Smadja, and M. Wood-Vasey, "Overview of the Nearby Supernova Factory", 2002SPIE.4836...61A

2. E. Pecontal, G. Aldering, G. Adam, P. Antilogus, P. Astier, Y. Copin, F. Henault, J.P. Lemonnier, P. Nugent, R. Pain, A. Pecontal, S. Perlmutter, R. Quimby, G. Smadja and M. Wood-Vasey, "The Nearby Supernova Factory", ESO, 2003fthp.conf..404P

3. R. Bacon, G. Adam, A. Baranne, G. Courtes, D. Dubet, J.P. Dubois, E. Emsellem, P. Ferruit, Y. Georgelin, G. Monnet, E. Pecontal, A. Rousset and F. Say, "3D spectrography at high spatial resolution. I. Concept and realization of the integral field spectrograph TIGER”, 1995A\&AS..113..347B 